\title{
BƯớC ĐẦU XÂY DỰNG THANG ĐÁNH GIÁ Đ!̣NH LƯợNG ĐỐI VớI ĐỀ ÁN \& BÁO CÁO KẾT QUẢ HOẠT ĐộNG CẢI TIẾN CHẤT LƯợNG
}

\author{
Đỗ Văn Niệm ', Đặng Thanh Tuấn'', Lê Thị Thu Thúy' \\ 1. Phòng Quản lý chất lượng Bệnh viện Nhi Đồng 1 \\ 2. Phòng Kếhoạch tổng hợp Bệnh viện Nhi Đồng 1
}

TÓM TẮT

Giới thiệu: Công cụ đánh giá tốt là điều kiện cân để hỗ trợ các nhóm cải tiến và đảm bảo chất lượng hoạt động cải tiến. Mục tiêu: Thiết kế mẫu đánh giá đề án và báo cáo kết quả cải tiến theo thang điểm định lượng. Phương pháp: Kết hợp tổng quan tài liệu với thảo luận nhóm và phỏng vấn chuyên gia để xây dựng thang đo, đánh giá giá trị nội dung và giá trị diễn đạt. Phân tích tỷ số giá trị nội dung (CVR) và điểm số ảnh hưởng (IIS) của từng mục để quyết định nội dung được giữ lại. Kết quả: Toàn bộ 22 mục của mẫu đánh giá đề án đạt yêu câu với CVR>0,6. IIS của các mục 1.6, 2.1b, 3.3 và 3.5 ở ngay dưới ngướng 1,5 đối với tiêu chí "quan trọng". Tương tự nhu" vậy ở mục 2.1c đối với tiêu chí "cân thiết". Đối với mẫu đánh giá báo cáo hoạt động cải tiến: Có 3 mục là 3.3, 3.4 và 3.5 không đạt yêu câuu CVR >0,49 và IIS > 1,5 ở tiêu chí "quan trọng" và "cân thiết" (mục 3.3 và 3.5). Những mục này không đảm bảo giá trị diễn đạt nhưng là nội dung bắt buộc trong chuẩn đánh giá của cơ quan quản lý nhà nước, nên được giữ lại trong biểu mẫu cuối cùng. Kết luận: Thang đánh giá đề án và báo cáo cải tiến đảm bảo yêu câuu cơ bản về giá trị nội dung nên có thể sử dụng. Cần nghiên cứu thêm để hoàn thiện giá trị diễn đạt và đánh giá thêm về giá trị cấu trúc.

Từ khóa: Cải tiến chất lượng, Thang đánh giá, Plan-Do-Check-Act (PDCA).

\section{ABSTRACT \\ DEVELOPING THE FIRST VERSION QUANTITATIVE SCALE IN EVALUATION OF QUALITY IMPROVEMENT PROJECTS AND REPORTS}

Do Van Niem, Dang Thanh Tuan, Le Thi Thu Thuy

Introduction: An appropriate evaluation tool is a necessary condition for supporting quality improvement teams \& ensuring the quality of initiative activities. Objectives: To design a quantitative scale for evaluation of quality improvement (QI) projects and reports. Methods: To integrate literature review with expert's focus-group discussion for developing evaluation scales and testing their content and face validities. Content validity ratio (CVR) and item impact score (IIS) for every item were analyzed to make decision on items retained. Results: All of 22 items from QI-project evaluation scale met the requirement of CVR > 0.6. IIS of items 1.6, 2.1b, 3.3 and 3.5 were just below the threshold of 1.5 on "importance" criterion. The similar thing was found for item $2.1 \mathrm{c}$ on "necessary" criterion. In QI-report evaluation scale, 3 items of 3.3, 3.4 and 3.5 did not meet requirements of CVR $>0.49$ and

Nhận bài: 15-12-2020; Chấp nhận: 10-2-2021

Người chịu trách nhiệm chính: Đỗ Văn Niệm

Địa chỉ: Bệnh viện Nhi Đồng 1 TP. HCM

(niemdv@nhidong.org.vn) 
IIS > 1.5 on "importance" and "necessary" criterion (item 3.3 and 3.5). Although three aforementioned items did not meet face validity criterion, they were still kept in the final scales because of mandatory requirements from the Vietnam authorities. Conclusion: The QI-project and QI-report evaluation scales met basic requirements of content validity for application in practice. Further studies are needed to improve face validity and test construct validity.

Keywords: Quality Improvement (QI), evaluation scale, Plan-Do-Check-Act (PDCA)

\section{1. ĐẶT VẤN ĐỀ}

Hoạt động cải tiến chất lượng đã phát triển trong nhiều năm qua từ khi Bộ $\mathrm{Y}$ tế triển khai Thông tư 19/2013/TT-BYT hướng dẫn thực hiện quản lý chất lượng bệnh viện. Trung bình có khoảng 20-25 đề án cải tiến chất lượng được triển khai tại Bệnh viện Nhi Đồng 1 mỗi năm. Một số biểu mẫu lượng giá chất lượng đề án và báo cáo hoạt động cải tiến đã được thiết kế và áp dụng tại Bệnh viện Nhi Đồng 1 (hiện đang áp dụng phiên bản 2.0 năm 2016), dựa trên khung cấu trúc báo cáo cải tiến SQUIRE 2.0 (Standards for Quality Improvement Reporting Excellence) $)^{(1)}$ và dựa trên quá trình thực hiện cải tiến theo tiếp cận PDCA. Thang đánh giá sơ bộ định lượng đã bước đầu thí điểm trong đánh giá đề án cải tiến đối với học viên tham dự khóa đào tạo cải tiến chất lượng nâng cao tại bệnh viện giai đoạn 2017-2019. Tuy nhiên, những công cụ này chưa đủ chi tiết, còn thiên hướng đánh giá định tính, chưa đồng bộ về nội dung ở các phần giữa đánh giá đề án với báo cáo cải tiến, chưa thống nhất với cấu trúc 3 phần chính theo hướng dẫn đánh giá sản phẩm sáng kiến của Hội đồng Thi đua khen thưởng Thành phố Hồ Chí Minh, và chưa được nghiên cứu đánh giá tính giá trị và độ tin cậy.

Công trình nghiên cứu cải tiến chất lượng là một trong những tiêu chuẩn quan trọng để đánh giá thi đua cá nhân và tập thể mỗi năm, cũng như nâng hạng viên chức và xét duyệt danh hiệu thi đua theo Luật Thi đua - khen thưởng (tương tự như một công trình nghiên cứu khoa học). Công cụ đánh giá hoạt động cải tiến có chất lượng tốt không những là yếu tố quan trọng giúp đảm bảo chất lượng hoạt động cải tiến, mà còn là điều kiện cần thiết để đảm bảo công bằng trong thi đua - khen thưởng.

Khung lý thuyết về sự thay đổi có 3 yếu tố tác động lẫn nhau quyết định khả năng thành công của hoạt động cải tiến trên thực tế, cũng như quá trình đánh giá đề án và báo cáo kết quả cải tiến. Đó là: 1) Điều gì cần thay đổi (WHAT), 2) Bối cảnh thay đổi (CONTEXT) và 3) Thay đổi bằng cách nào (HOW). Chủ đề (nội dung) cải tiến, phương pháp triển khai có tốt hay không cần phải được đặt vào bối cảnh cụ thể. Việc đánh giá cần phải được xem xét tổng thể trên cơ sở đảm bảo sự cân bằng giữa các yếu tố. Mô hình MUSIQ (Model for Understanging Success in Quality) là một trong những khung cấu trúc cơ bản để tiếp cận đánh giá hoạt động cải tiến [2]. Hình 1 trình bày tóm lược sự tương tác giữa các yếu tố trên và khung cấu trúc để thực hiện đánh giá đề án cải tiến.

Mặc dù có nhiều mô hình tiếp cận đánh giá sự thành công của đề án và hướng dẫn bố cục, nội dung báo cáo cải tiến như MUSIQ,[2] SQUIRE 2.0[1] đã được giới thiệu, nhóm nghiên cứu chưa tìm thấy bất kỳ thang đánh giá đề án và báo cáo cải tiến theo thang điểm định lượng nào. Hầu hết các hướng dẫn đánh giá tập trung vào phương pháp phân tích dữ liệu đánh giá hiệu quả cải tiến dựa trên thiết kế nghiên cứu hoặc tiếp cận theo quá trình (Leviton 2010[3], Healthier 2012[2], Coly 2017[4], Gareth 2018[5]). Gareth và cộng sự (2018) đề xuất tiếp cận lượng giá hoạt động cải tiến khá chi tiết, dựa trên cây quyết định và mô hình khung logic để đánh giá hoạt động cải tiến theo tiếp cận quá trình, tương thích với hướng dẫn báo cáo cải tiến SQUIRE 2.0, nhưng vẫn chưa đưa ra thang đánh giá định lượng. Biểu mẫu đánh giá dự án định lượng do Đỗ Huân (2016) giới thiệu[6], được Việt hóa và áp dụng tại Việt Nam dựa trên nguồn hướng dẫn của Cộng đồng chung châu Âu, nhưng nó được phát triển đặc thù cho dự án triển khai đào tạo cộng đồng, chứ không phải sáng kiến cải tiến.

Vì vậy, nhóm nghiên cứu thiết kế biểu mẫu lượng giá mới (phiên bản 3.0), đánh giá giá trị nội 
dung và mức độ ảnh hưởng của các mục chi tiết trong thang đo, làm cơ sở để lượng giá đề án và báo cáo cải tiến tốt hơn, nhằm góp phần thúc đẩy và nâng cao chất lượng hoạt động cải tiến chất lượng tại các bệnh viện hiện nay.

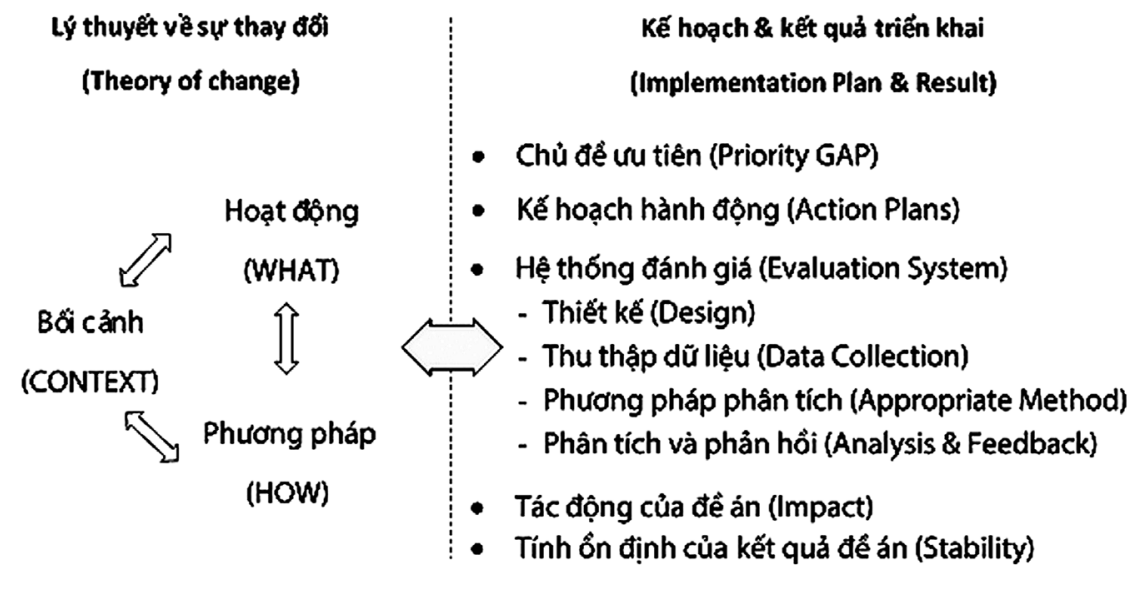

Hình 1. Mối quan hệ giữa lý thuyết về sự thay đổi và hoạt động cải tiến

\section{2. ĐỐI TƯợNG VÀ PHƯơNG PHÁP}

2.1. Đối tượng khảo sát: Chọn các chuyên gia công tác tại bệnh viện làm việc ở các bộ phận có liên quan đến hoạt động cải tiến, thường tham gia các hoạt động đánh giá đề án và báo cáo kết quả hoạt động cải tiến chất lượng. Người trực tiếp thiết kế biểu mẫu và thang đo không tham gia đánh giá. Cần lưu ý, đối tượng khảo sát trong nghiên cứu này vừa đóng vai trò là chuyên gia trong lính vực cải tiến, đồng thời cũng là đối tượng sử dụng mẫu đánh giá sau này.

\subsection{Phương pháp}

2.2.1. Thiết kế: Nghiên cứu định tính dựa trên tổng quan tài liệu, thảo luận nhóm kết hợp với phỏng vấn chuyên gia bằng mẫu câu hỏi tự điền.

2.2.2. Thang đo: Tham khảo khung cấu trúc đánh giá dự án can thiệp về đào tạo của Cộng đồng chung châu Âu, được Việt hóa và áp dụng trong các dự án can thiệp về đào tạo, được tác giả Đỗ Huân giới thiệu[6], các nội dung chi tiết được tổng quan từ các biểu mẫu đánh giá hiện áp dụng tại bệnh viện và khung cấu trúc báo cáo hoạt động cải tiến SQUIRE 2.0[1], tiếp cận đánh giá đề án cải tiến MUSIQ[2] để thiết kế khung cấu trúc thang đánh gồm 3 phần chính (đối với đề án gồm: 1 ) sự phù

\section{Mục tiêu}

Phát triển mẫu lượng giá đề án cải tiến chất lượng thực hiện theo tiếp cận Plan-Do-Check-Act và báo cáo kết quả hoạt động cải tiến theo thang điểm định lượng.

Kế hoạch \& kết quả triển khai

(Implementation Plan \& Result)

hợp bối cảnh và phương pháp tiếp cận, 2) phương pháp đánh giá tác động của đề án, 3) tiềm năng của đề án, nguồn lực $\&$ tiến độ; đối với báo cáo cải tiến gồm: 1) sự phù hợp bối cảnh và phương pháp tiếp cận, 2) phương pháp đánh giá kết quả \& tác động của đề án, 3) tính bền vững của kết quả đề án), gồm 22 mục đối với đề án và 23 thành phần đối với báo cáo cải tiến. Mẫu lượng giá đề án và báo cáo kết quả hoạt động cải tiến được thiết kế theo thang điểm bách phân (100 điểm) nhằm dễ quy đổi thang đo trong quá trình sử dụng. Các mục quan trọng hoặc những mục đánh giá có mức chi tiết cao hơn được điều chỉnh trọng số (nhân hoặc chia 2), nhằm đảm bảo tính cân đối ở 3 phần của thang đo, với cơ cấu điểm các phần theo thứ tự là 35-35-30 đối với đề án và 30-40-30 đối với báo cáo kết quả cải tiến. Sử dụng kết quả này để thiết kế biểu mẫu đánh giá tương ứng, đồng thời thiết kế bộ câu hỏi khảo sát ý kiến chuyên gia, đánh giá đồng thời cả 2 tiêu chí "cần thiết" và "quan trọng" theo thang điểm Likert 1-5. Mẫu đánh giá đề án, báo cáo kết quả cải tiến phiên bản 3.1 được trình bày ở phần phụ lục.

2.2.3. Khảo sát \& chuẩn bị dữ liệu phân tích: Tổ chức thảo luận nhóm chuyên gia để làm rõ mục đích, nội dung mẫu khảo sát trước khi các chuyên 
gia chấm điểm khảo sát cho từng nội dung chi tiết. Dựa trên kết quả khảo sát để mã hóa thành thang Likert 3 khoảng để tính tỷ số giá trị nội dung CVR (Content Validity Ratio). Nếu người trả lời nội dung "cần thiết" dưới 3 điểm sẽ chấm điểm 1 (Không cần thiết). Nếu trả lời "cần thiết" từ 3 điểm trở lên, nhưng tiêu chí "quan trọng" dưới 3 sẽ chấm điểm 2 (cần thiết nhưng không quan trọng). Nếu cả 3 tiêu chí đều được đánh giá từ 3 điểm trở lên sẽ cho điểm 3 (cần thiết và quan trọng).

2.2.4. Phân tích dữ liệu:Tỷ số giá trị nội dung (CVR) và điểm số ảnh hưởng được tính cho từng mục (IIS: Item Impact Score), từng phần (PIS: Partial Impact Score) hoặc toàn bộ thang đo (TIS: Total Impact Score). Điểm số ảnh hưởng sẽ thể hiện giá trị diễn đạt (Face validity) cho nội dung tương ứng. CVR được tính theo công thức: $\mathrm{CVR}=(\mathrm{Ne}-\mathrm{N} / 2) /(\mathrm{N} / 2)$. Trong đó: Ne: Số người chọn "cần thiết và quan trọng"; $\mathrm{N}$ : Tổng số người trả lời mục hỏi; Ngưỡng tối thiểu giữ lại (Cut-off): CVR > 0,49.[7,8] Ngưỡng chấp nhận CVR điều chỉnh nếu số chuyên gia tham gia phỏng vấn là 10 , cần đạt $>0,62$ để đảm bảo có ý nghĩa thống kê với $p<0,05[7]$. IIS được tính bằng [phần trăm người trả lời mức 4 hoặc 5] $x$ [điểm trung bình của mục hỏi]. Ngưỡng chấp nhận là IIS > 1,5 (50\% x 3) [8]. PIS và TIS được tính tương tự như IIS, nhưng áp dụng cho toàn bộ các mục ở mỗi phần hoặc toàn bộ thang đo. Phân tích dữ liệu thực hiện trên ứng dụng Microsoft $\odot$ Excel 2016, sử dụng kết hợp các hàm IF, COUNTIF, SUM, AVERAGE để tính các chỉ số đánh giá chất lượng thang đo về giá trị nội dung.

Y đức nghiên cứu: Đề tài là một sản phẩm trong nghiên cứu cải tiến “Hoạt động hỗ trợ kỹ thuật và thúc đẩy phong trào chất lượng cấp khoa, phòng tại Bệnh viện Nhi Đồng 1" được Hội đồng thẩm định thông qua ngày 06-11-2019 và Giám đốc bệnh viện cho phép thực hiện tại Quyết định số 3106/QĐ-BVNĐ1 ngày 15-11-2019.

\section{KẾT QUẢ}

Có 10 chuyên gia đủ tiêu chí chọn lựa tham gia khảo sát. Trong đó có 3 bác sĩ, 3 điều dưỡng và 4 cử nhân y tế công cộng từ phòng Quản lý chất lượng, Kế hoạch tổng hợp, Chỉ đạo tuyến và Điều dưỡng; là những người tham gia tư vấn cho các nhóm cải tiến, đánh giá đề án cải tiến và báo cáo kết quả hoạt động cải tiến chất lượng tại bệnh viện. Người trực tiếp thực hiện tổng quan tài liệu, thiết kế mẫu khảo sát không tham gia chấm điểm đánh giá.

Tỷ số giá trị nội dung đạt yêu cầu $100 \%$ mục chi tiết đối với mấu đánh giá đề án cải tiến. Điểm thấp nhất là 0,6 ở mục 3.3 (nhóm cải tiến có đủ người đại diện và năng lực thực hiện). Kết quả này tương tự đối với mẫu đánh giá báo cáo cải tiến, trừ 3 mục từ 3.4 đến 3.6 (nhân rộng nơi khác, đóng góp cho cơ quan quản lý, giải thưởng hoặc quyền sở hữu/quyền tác giả) không đạt yêu cầu. Các nội dung này liên quan đến bằng chứng nhân rộng, báo cáo cơ quan quản lý và đạt giải thưởng hoặc bằng chứng nhận quyền tác giả/sáng chế. Đây là 3 nội dung quan trọng đối với sản phẩm sáng kiến/cải tiến chất lượng nhưng khó đạt yêu cầu. Mục có giá trị CVR thấp nhất là 0 , tương ứng mức $50 \%$ chuyên gia đồng ý về nội dung. Giá trị CVR cho từng mục trình bày tại bảng 1.

Bảng 1. Giá trị nội dung (CVR) của các mục thành phần trong thang đánh giá

Thang đánh giá đề án cải tiến

\begin{tabular}{|c|c|c|c|c|c|}
\hline Câu & CVR & Câu & CVR & Câu & CVR \\
\hline 1.1 & 1 & $2.1 \mathrm{a}$ & 1 & 3.1 & 1 \\
\hline 1.2 & 1 & $2.1 \mathrm{~b}$ & 1 & 3.2 & 1 \\
\hline 1.3 & 1 & $2.1 \mathrm{c}$ & 0,8 & 3.3 & 0,6 \\
\hline 1.4 & 1 & $2.1 \mathrm{~d}$ & 1 & 3.4 & 0,8 \\
\hline 1.5 & 1 & $2.2 \mathrm{a}$ & 1 & 3.5 & 0,8 \\
\hline 1.6 & 0,8 & $2.2 \mathrm{~b}$ & 1 & 3.6 & 1 \\
\hline & & $2.2 \mathrm{c}$ & 1 & & \\
\hline & & $2.2 \mathrm{~d}$ & 1 & & \\
\hline & & $2.3 \mathrm{a}$ & 1 & & \\
\hline & & $2.3 \mathrm{~b}$ & 1 & & \\
\hline & & & & & \\
\hline & & & & & \\
\hline & & & & & \\
\hline
\end{tabular}

Thang đánh giá báo cáo cải tiến

\begin{tabular}{|c|c|c|c|c|c|}
\hline Câu & CVR & Câu & CVR & Câu & CVR \\
\hline 1.1 & 1 & $2.1 \mathrm{a}$ & 1 & 3.1 & 1 \\
\hline 1.2 & 1 & $2.1 \mathrm{~b}$ & 1 & 3.2 & 1 \\
\hline 1.3 & 1 & $2.1 \mathrm{c}$ & 0,8 & 3.3 & 0,6 \\
\hline 1.4 & 1 & & & 3.4 & 0 \\
\hline 1.5 & 1 & $2.2 \mathrm{a}$ & 1 & 3.5 & 0,4 \\
\hline 1.6 & 0,8 & $2.2 \mathrm{~b}$ & 1 & 3.6 & 0 \\
\hline & & $2.2 \mathrm{c}$ & 1 & & \\
\hline & & $2.2 \mathrm{~d}$ & 1 & & \\
\hline & & $2.3 \mathrm{a}$ & 1 & & \\
\hline & & $2.3 \mathrm{~b}$ & 1 & & \\
\hline & & $2.3 \mathrm{c}$ & 1 & & \\
\hline & & $2.3 \mathrm{~d}$ & 1 & & \\
\hline & & $2.3 \mathrm{e}$ & 1 & & \\
\hline
\end{tabular}


Điểm số ảnh hưởng toàn bộ thang lượng giá (TIS) của đề án và báo cáo cải tiến ở tiêu chí"cần thiết" cao hơn tiêu chí "quan trọng". Điểm số ảnh hưởng từng phần và toàn bộ thang đều đạt yêu cầu > 1,5. Trong đó điểm phần 3 là thấp nhất cho cả 2 thang đánh giá đề án và báo cáo cải tiến ở cả 2 tiêu chí (bảng 2 ).

Bảng 2. Điểm số ảnh hưởng của thang đo theo tiêu chí "cần thiết" \& "quan trọng"

\begin{tabular}{|c|c|c|c|}
\hline & $\begin{array}{c}\text { Tần suất }[4,5] \\
\text { (Frequency) }\end{array}$ & $\begin{array}{l}\text { Trung bình } \\
\text { (Importance) }\end{array}$ & IIS/PIS/TIS \\
\hline \multicolumn{4}{|l|}{ Thang đánh giá đề án cải tiến: } \\
\hline \multicolumn{4}{|l|}{ Tiêu chí "cần thiết" } \\
\hline Sự phù hợp và phương pháp tiếp cận & 0,88 & 4,5 & 3,99 \\
\hline Phương pháp đánh giá tác động & 0,84 & 4,2 & 3,56 \\
\hline Tiềm năng, nguồn lực \& tiến độ & 0,77 & 4,2 & 3,18 \\
\hline Toàn bộ & 0,83 & 4,3 & 3,57 \\
\hline \multicolumn{4}{|l|}{ Tiêu chí "quan trọng" } \\
\hline Sự phù hợp và phương pháp tiếp cận & 0,8 & 4,3 & 3,44 \\
\hline Phương pháp đánh giá \& tác động & 0,71 & 4 & 2,86 \\
\hline Tính bền vững của kết quả đề án & 0,6 & 3,9 & 2,33 \\
\hline Toàn bộ & 0,7 & 4,1 & 2,86 \\
\hline \multicolumn{4}{|l|}{ Thang đánh giá báo cáo cải tiến: } \\
\hline \multicolumn{4}{|l|}{ Tiêu chí "cần thiết" } \\
\hline Sự phù hợp và phương pháp tiếp cận & 0,8 & 4,5 & 3,63 \\
\hline Phương pháp đánh giá tác động & 0,92 & 4,4 & 4,06 \\
\hline Tiềm năng, nguồn lực \& tiến độ & 0,67 & 3,9 & 2,6 \\
\hline Toàn bộ & 0,85 & 4,3 & 3,68 \\
\hline \multicolumn{4}{|l|}{ Tiêu chí "quan trọng" } \\
\hline Sự phù hợp và phương pháp tiếp cận & 0,87 & 4,3 & 3,76 \\
\hline Phương pháp đánh giá \& tác động & 0,79 & 4,2 & 3,35 \\
\hline Tính bền vững của kết quả đề án & 0,53 & 3,7 & 1,95 \\
\hline Toàn bộ & 0,75 & 4,1 & 3,07 \\
\hline
\end{tabular}

Chỉ số IIS đối với biểu mẫu lượng giá đề án không đạt yêu cầu ở mục 2.1c (có kế hoạch đánh giá ảnh hưởng đến các bộ phận có liên quan nhưng nằm ngoài phạm vi can thiệp của đề án) đối với tiêu chí "cần thiết" (IIS=1,36) nhưng đạt yêu cầu ở tiêu chí "quan trọng" (IIS =2,16). Các mục 1.6 (khả năng tăng giá trị cho tổ chức), 2.1b (kế hoạch so sánh tương quan chỉ số quá trình và kết quả), 3.3 (nhóm cải tiến đủ thành phần và năng lực thực hiện), 3.5 (đủ thẩm quyền và khả năng huy động nguồn lực thực hiện đề án) không đạt yêu cầu ở tiêu chí "quan trọng" (IIS theo thứ tự là 1,4 - 1,4 - 1,32 - 1,4) nhưng đạt yêu cầu ở tiêu chí "cần thiết" (IIS tương ứng theo tứ tự là 2,28 $3,01-2,66-2,8)$.

Chỉ số IIS đối với biểu mẫu lượng giá báo cáo cải tiến không đạt yêu cầu ở mục 3.4 (biện pháp nhân rộng cơ sở y tế khác) và 3.6 (đạt giải thưởng chuyên ngành hoặc đăng ký sáng chế/quyền sở hữu) đối với tiêu chí "cần thiết" và "quan trọng" (IIS tương ứng theo thứ tự là $1,44-1,16$ và 0,9 0,56). Mục 3.5 (đóng góp giải pháp cho cơ quan quản lý) đạt yêu cầu tiêu chí "cần thiết" (IIS=1,85) 
nhưng không đạt yêu cầu ở tiêu chí "quan trọng" (IIS=0,96).

Các thành phần chi tiết của nội dung có điều chỉnh bằng trọng số (nhân đôi hoặc chia đôi) trong mẫu dự thảo đều có điểm đánh giá nội dung và mức ảnh hưởng tốt, đạt mức điểm tuyệt đối hoặc cao gần mức tuyệt đối.

Các ý kiến góp ý chi tiết chủ yếu tập trung nêu các khó khăn của các nhóm cải tiến trong quá trình thực hiện, không bổ sung thêm nội dung cần đánh giá của dự thảo biểu mẫu lượng giá.

\section{BÀN LUẬN VÀ KẾT LUẬN}

Cấu trúc mẫu đánh giá được thiết kế 3 phần phù hợp với tiếp cận theo quá trình trong quản lý chất lượng, khung pháp lý hướng dẫn đánh giá sản phẩm sáng kiến theo Hướng dẫn số 29/ HD-HĐXCNSKCTP của Hội đồng xét duyệt sáng kiến Thành phố Hồ Chí Minh năm 2018, đảm bảo sự phù hợp của yếu tố bối cảnh (ưu tiên và khả thi), phương pháp đánh giá và tác động của hoạt động trong đề án, tiềm năng và sự bền vững của kết quả đề án. Cấu trúc mẫu đánh giá này tương đồng với hướng dẫn báo cáo kết quả cải tiến SQUIRE 2.0[1].

Hầu hết các mục chi tiết của cả 3 cấu phần trong dự thảo mẫu lượng giá chất lượng đề án và báo cáo cải tiến đều đạt yêu cầu về tỷ số giá trị nội dung và điểm số ảnh hưởng. Không có ý kiến bổ sung, điều chỉnh cấu trúc của mẫu lượng giá. Điều này chứng tỏ dự thảo mẫu đánh giá đảm bảo những nội dung cốt lỗi cần đánh giá.

Các tiểu mục chi tiết không đạt yêu cầu ở chỉ số ảnh hưởng của 1 trong 2 tiêu chí "cần thiết" hoặc "quan trọng" nhưng là nội dung rất cần thiết nên nếu loại bỏ thì cần phải thay thế bằng tiểu mục có giá trị tương đương. Tuy nhiên, những thành phần tương đương chưa có trong biểu mẫu nên chúng cần được điều chỉnh nhằm cải thiện giá trị diễn đạt, mà không nên loại bỏ khỏi mẫu đánh giá.

Đối với mẫu lượng giá đề án, mục 1.6 là giá trị của đề án đối với tổ chức, mục $2.1 \mathrm{~b}$ đề cập 2 nhóm chỉ số đánh giá chủ yếu (quá trình và kết quả), mục $2.1 \mathrm{c}$ là chỉ số cân bằng, mục 3.3 giúp đảm bảo người đại diện đủ am hiểu về quá trình cải tiến và năng lực nhóm can thiệp, mục 3.5 liên quan tính khả thi của đề án (khả năng huy động nguồn lực và tổ chức thực hiện). Những nội dung này đều nằm trong 25 nội dung cốt lõi theo mô hình đánh giá MUSIQ và không có nội dung chồng lấn ở các mục khác của biểu mẫu[2]. Vi vậy, nhóm nghiên cứu quyết định tiếp tục giữ lại các mục này, nhưng cần tiếp tục nghiên cứu hiệu chỉnh về từ ngữ để hoàn thiện giá trị diễn đạt, nhằm đảm bảo chất lượng điểm số đánh giá, chứ không thể loại bỏ mà không bổ sung nội dung thay thế tương đồng về nội dung.

Một sản phẩm cải tiến có khả năng nhân rộng ở cơ sở khác (phạm vi ảnh hưởng rộng), đạt giá trị khoa học được công nhận thông qua giải thưởng khoa học hoặc được cấp bằng sáng chế/quyền sở hữu trí tuệ, hoặc đóng góp về giải pháp đối với cơ quan quản lý nhà nước chứng tỏ hoạt động cải tiến có giá trị cao nên không có lý do thực tiễn ủng hộ việc loại bỏ các thành phần này ra khỏi mẫu lượng giá báo cáo kết quả cải tiến (nếu dựa vào ý kiến đánh giá theo thang điểm của chuyên gia trong nghiên cứu này). Hầu hết ý kiến góp ý chi tiết cho thấy nội dung này khó đạt đối với hầu hết cải tiến có quy mô nhỏ ở cơ sở. Đây có thể là lý do các chuyên gia đánh giá thấp vai trò của các thành phần này trong biểu mẫu lượng giá dẫn đến chưa đạt giá trị diễn đạt ở mác mục từ 3.3 đến 3.5 trong mẫu lượng giá báo cáo cải tiến. Việc giữ lại các thành phần chi tiết này không những không làm mất đi cơ hội được nghiệm thu, mà còn có giá trị phân loại chất lượng đối với các báo cáo cải tiến. Bởi vì, nếu hoàn thành tốt các nội dung khác thì đề tài nghiên cứu cải tiến vẫn có khả năng đạt $85 \%$ điểm số, đủ điều kiện để xếp loại tốt với ngưỡng chấp nhận 80 điểm theo QT/Thủ tục Cải tiến chất lượng hiện hành của bệnh viện. Những đề tài đạt được $15 \%$ số điểm ở phần này, nghĩa là đề tài được kiểm chứng giá trị 
ứng dụng thực tiễn ở mức độ cao hơn nên xứng đáng có điểm đánh giá cao hơn. Vì vậy, nhóm quyết định giữa lại các thành phần này và tiếp tục nghiên cứu hoàn chỉnh về từ ngữ, nhằm đảm bảo giá trị diễn đạt trong quá trình sử dụng. Vi vậy, nhóm tham gia đánh giá đề án và báo cáo cải tiến cần được tập huấn đầy đủ, đảm bảo họ hiểu thống nhất nội dung đánh giá, nhằm đảm bảo chất lượng của điểm số đánh giá.

Điểm yếu của nghiên cứu này là số chuyên gia được phỏng vấn còn ít, chưa đánh giá ở nhiều bệnh viện nhằm đảm bảo tính đa dạng của bối cảnh. Do hạn chế về nguồn lực và thời gian thực hiện nên nhóm nghiên cứu áp dụng khảo sát 1 lần và mã hóa lại biến theo thang đo phù hợp để tính CVR nên có thể ảnh hưởng đến độ chính xác của kết quả nghiên cứu. Đồng thời, thang đo chỉ bước đầu được đánh giá về giá trị nội dung và giá trị diễn đạt, chưa đánh giá đầy đủ các tiêu chuẩn khác trong quá trình phát triển thang đo như đánh giá độ tin cậy và giá trị cấu trúc.

Tóm lại, biểu mẫu lượng giá đề án cải tiến theo tiếp cận PDCA và báo cáo cải tiến (phiên bản 3.1, đã hiệu chỉnh từ ngữ sau nghiên cứu) có 3 thành phần chính với 22 nội dung (đối với đề án) và 23 nội dung (đối với báo cáo kết quả), theo thang điểm bách phân, đảm bảo yêu cầu cơ bản về giá trị nội dung, có thể sử dụng được trong thực hành. Cần nghiên cứu thêm để hiệu chỉnh về từ ngữ và tập huấn cho nhóm đánh giá, nhằm hoàn thiện giá trị diễn đạt của các thành phần trong thang đo, đảm bảo chất lượng điểm số đánh giá các đề án và báo cáo cải tiến. Việc thực hiện nghiên cứu định lượng với cỡ mẫu lớn hơn, để đánh giá giá trị cấu trúc và tính bất biến theo bối cảnh, có thể cần thiết nhằm đảm bảo chất lượng và độ ổn định của thang đo.

\section{TÀI LIỆU THAM KHẢO}

1. Goodman D, Ogrinc G, Davies L et al (2016). Explanation and elaboration of the SQUIRE (Standards for Quality Improvement Reporting Excellence) Guidelines, V. 2.0: examples of SQUIRE elements in the healthcare improvement literature. BMJ Qual Saf; 25:7

2. Heather C Kaplan, Lloyd P Provost, Craig M Froehle, Peter A Margolis (2012). The Model for Understanding Success in Quality (MUSIQ): building a theory of context in healthcare quality improvement. BMJ Qual Saf; 21:13e20. doi:10.1136/bmjqs-2011-000010.

3. Leviton LC, Khan LK, Rog D et al (2010). Evaluability assessment to improve public health policies, programs, and practices. Annu Rev Public Health; 31:213-33.

4. Coly A, Parry G (2017). Evaluating Complex Health Interventions: A Guide to Rigorous Research Designs. Academy Health.

http://www.academyhealth.org/evaluationguide.

5. Gareth Parry, Astou Coly, Don Goldmann, Alexander K. Rowe, Vijay Chattu Loiudice, Mihajlo Rabrenovic, and Bejoy Nambiar (2018). Practical recommendations for the evaluation of improvement initiatives. International Journal for Quality in Health Care, 30 (S1), 29-36. doi: 10.1093/intqhc/mzy021.

6. Đỗ Huân (2016). Phụ lục 12: Mẫu đánh giá đề án, trong: Nhà đào tạo sành sỏi. Nxb Lao động, tr. 446-449.

7. C. H. Lawshe (1975). A quantitative approach to content validity. Personel Psychology, 28: 563-575.

8. Zamanzadeh V, Ghahramanian A, Rassouli M, Abbaszadeh A, et al (2015). Design and Implementation Content Validity Study: development of an instrument for measuring Patient-Centered Communication. Journal of Caring Sciences, 4(2): 165-178. DOI: 10.15171/jcs.2015.107. 